\title{
A Rare Case of Single Right Coronary Artery with Absent Left Main Coronary Artery
}

\author{
Aidan (Jia Sheng) Yu' ${ }^{1 *}$, Matthew Rowe'2, John Atherton', Arun Dahiya' \\ 'Royal Brisbane and Women's Hospital, Australia \\ ${ }^{2}$ Princess Alexandra Hospital, Australia
}

Article Info

\section{Article Notes}

Received: February 16, 2019

Accepted: March 11, 2019

\section{${ }^{*}$ Correspondence:}

Dr. Aidan (Jia Sheng) Yu, Royal Brisbane and Women's Hospital, Butterfield Street and Bowen Bridge Road, Herston,

Queensland, 4029, Australia; Email: aidanjsy@gmail.com.

(c) 2019 Yu A. This article is distributed under the terms of the Creative Commons Attribution 4.0 International License.

\section{Keywords}

Single coronary anomaly

Absent LMCA

Super-dominant RCA

\section{Abstract}

A 54-year-old female presented with chest pain and was noted to have new T-wave inversion on her electrocardiograph (ECG) in leads V2-V6. The patient has a past history of recurrent DVT/PE and recently had a subtotal colectomy for ulcerative colitis. Serial troponin I measurements were normal $(<0.040 \mu \mathrm{g} / \mathrm{l})$. Transthoracic echocardiogram revealed an impaired left ventricular ejection fraction of $35-40 \%$ with hypokinesis within the lateral and apical segments. A coronary angiogram was performed and the left main coronary artery (LMCA) could not be located despite cuspal injections, ascending aortogram and left ventriculogram. A large, dominant right coronary artery (RCA) was visualised which passed around the left ventricular apex to the area normally supplied by the obtuse marginal (OM), diagonal and distal left anterior descending (LAD) arteries. Computed tomography coronary angiography (CTCA) revealed a superdominant RCA supplying the LV apex and lateral wall with no LMCA coming off the left coronary cusp. The left circumflex (LCX) was small and arose from a conus branch with a separate origin to the RCA. No significant stenosis was visualised on CTCA. The T-wave changes were deemed to be secondary to physiological stress/supply-demand ischaemia particularly at the distal end of the RCA, where it supplied the LAD. The patient was commenced on metoprolol $25 \mathrm{mg}$ b.d. and continued warfarin on discharge.

Single RCA with absent LMCA is extremely rare with only a few isolated cases reported in the literature. Use of CTCA in combination with coronary angiography was useful in defining this unusual anatomy and excluding a haemodynamically significant lesion. This anomaly is a variation to the typical Lipton subtypes and may represent a new subtype not previously described before.

\section{Case Details}

A 54-year-old female presented with chest pain and was noted to have new T-wave inversion on electrocardiograph (ECG) in leads V2-V6. Her past medical history is significant for recurrent DVT/PE, rheumatic fever, ulcerative colitis and ampullary neuroendocrine tumour. This patient was recently admitted for a subtotal colectomy and ileostomy for ulcerative colitis. Serial troponin I measurements were normal $(<0.040 \mu \mathrm{g} / \mathrm{l})$.

A transthoracic echocardiogram was performed and it revealed impairment of the left ventricular systolic function with an ejection fraction of $35-40 \%$. Hypokinesis was present in the lateral and apical segments. The initial presentation was concerning of Takotsubo cardiomyopathy or myocardial ischaemia. This was further investigated with a coronary angiogram to evaluate for any coronary artery disease. A large, dominant right coronary artery (RCA) was visualised and it passed around the left ventricular apex to the area 
normally supplied by the obtuse marginal (OM), diagonal and distal left anterior descending (LAD) arteries (Figure $1 \mathrm{~A}, \mathrm{~B})$. The left main coronary artery (LMCA) could not be located despite cuspal injections, ascending aortogram and left ventriculogram (Figure 1C). Further investigation with a computed tomography coronary angiography (CTCA) revealed a superdominant RCA supplying the LV apex and lateral wall with no LMCA coming off the left coronary cusp (Figure 2A). The distal branch of RCA continued as posterior descending artery (PDA) to supply the LAD territory retrogradely. The left circumflex was small and arose from a conus branch with a separate origin to the RCA. No significant stenosis was visualised on CTCA.

A cardiac MRI (CMRI) was also performed which revealed subtle subendocardial enhancement and fibrosis in the mid-anterior wall. This was thought to be likely related to chronic subendocardial ischaemia in the context of patient's single coronary artery (Figure 2B). In addition, left ventricular systolic function was preserved

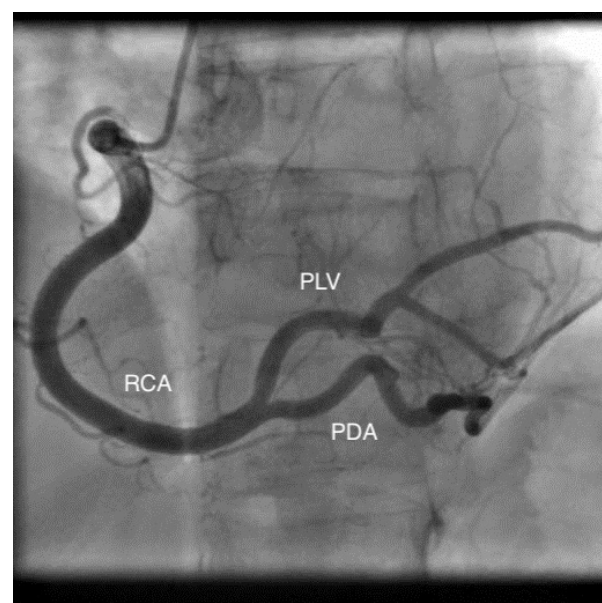

Figure $\mathbf{1}(\mathrm{A})$. Coronary angiogram (AP) showing RCA with its branches PLV and PDA suppling lateral LV wall.

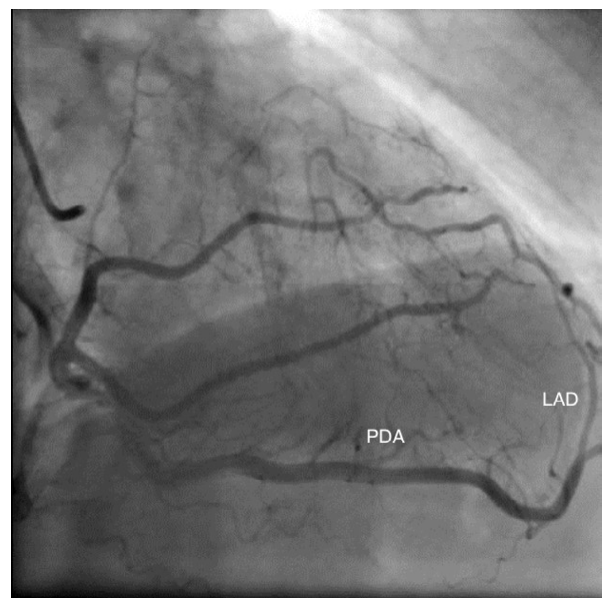

Figure 1(B). Coronary angiogram (RAO) showing distal branches of RCA supplying LAD territory.

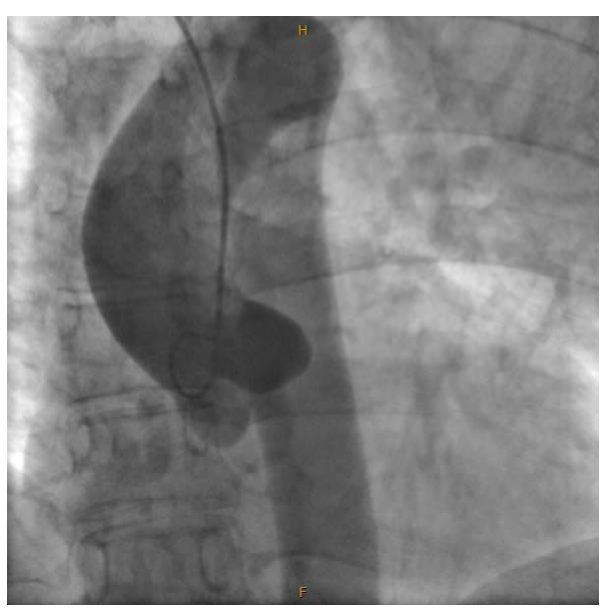

Figure $\mathbf{1}(\mathrm{C})$. Coronary angiogram showing no LMCA arising from the left coronary cusp.

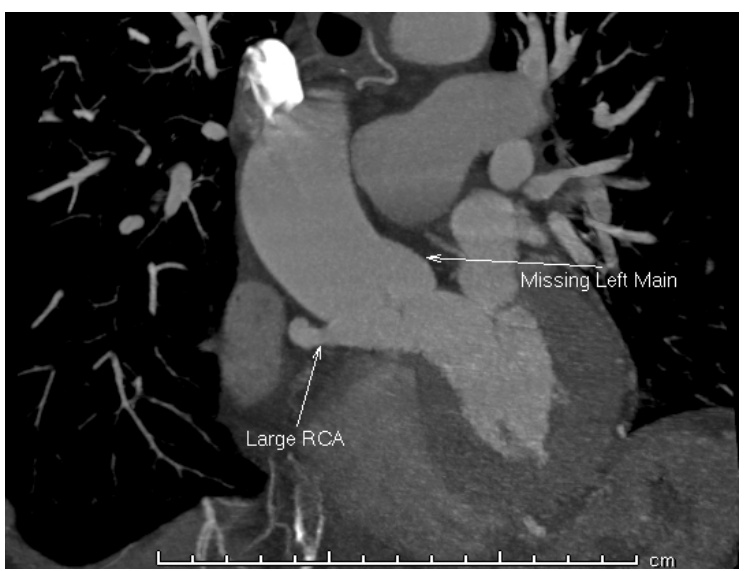

Figure 2(A). CTCA showing a prominent RCA and absent LMCA.

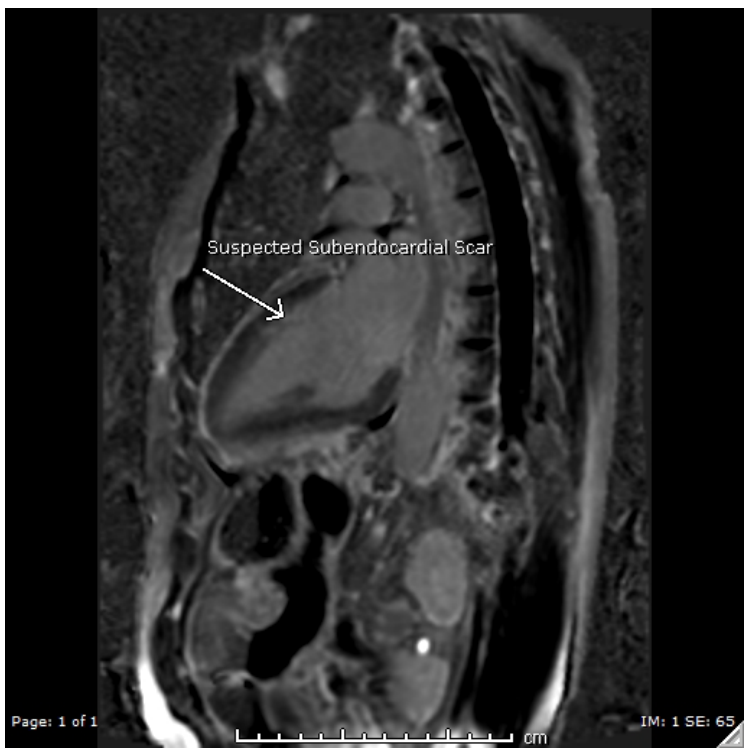

Figure 2(B). CMRI showing subtle subendocardial scar.

on CMRI with left ventricular ejection fraction measuring at $58 \%$. 
The T-wave changes were deemed to be secondary to physiological stress/supply-demand ischaemia particularly at the distal end of the RCA, where it supplied the LAD. The patient was commenced on metoprolol $25 \mathrm{mg}$ b.d. and continued warfarin on discharge.

\section{Discussion}

Coronary artery anomalies are extremely rare with single right coronary artery being the rarest at an incidence of $0.0008 \% 1$. These can occur in isolation or with other congenital heart diseases, most commonly valvular anomalies. Anomalous coronary arteries in the absence of other congenital heart diseases are not associated with an increased risk of atherosclerotic development or decreased life expectancy ${ }^{2,3}$. However, patients with SCA who present with acute coronary syndrome have a higher risk of interventional complications ${ }^{2}$. SCA may contribute to angina as patients can experience transient ischaemia particularly in stressful conditions where supply is not able to meet its demand. In addition, minor proximal vessel disease in SCA may also impact on distal coronary circulation $^{3}$. This was well correlated in our patient as the subendocardial enhancement and fibrosis seen in the anterior wall were reflective of chronic subendocardial ischaemia where the single right coronary artery was unable to provide sufficient oxygen during conditions of increased demand.

Lipton's categorisation of single coronary anomalies is the most universally used classification system. A single dominant RCA would be defined as R-I type. It is further classified as type A (Anterior), B (Between), P (Posterior), $\mathrm{S}$ (Septal) or C (Combined) depending on the course of the transverse trunk. In R-I, the RCA originates from the right coronary sinus and follows the course of a normal RCA, giving off PDA and continues in the left atrioventricular groove before giving off posterolateral left ventricular branches. The distal branch of RCA then continues in the atrioventricular groove as LCx prior to reaching the anterior basal surface of the heart where it gives off LAD as its terminal branch ${ }^{3}$. The coronary anatomy, in this case, differs from the typical R-I subtype as the RCA does not continue to form LCx and LAD as its terminal branches. Instead, the distal branches of RCA extend to the anterolateral wall and apical segments to supply the distal LAD, OM and diagonal territories. In Lipton R-II, the single RCA would cross at the base of the heart as a large transverse trunk before branching off as LAD and $\mathrm{LCx}^{3}$. The small hypoplastic LCx in our case arises from a conus branch arising off the right coronary cusp separate to the RCA. This anomaly is a variation to the typical Lipton subtypes and may represent a new subtype not previously described before.

Occlusion of LMCA and left coronary artery had to be excluded prior to making a diagnosis of single RCA. The presence of a blind left coronary sinus in CTCA favoured the absence of LMCA rather than an atherosclerotic LMCA occlusion. It would also be unusual to have a superdominant RCA supplying the LAD and LCx territories if the LMCA was present. Notably, the patient's CMRI showed a preserved left ventricular systolic function with an ejection fraction of $58 \%$ which suggested intact coronary circulation. However, it is important to note that the absence of a coronary artery on contrast imaging does not confirm its absence. The superdominant RCA visualised along with subtle subendocardial ischaemia seen on CMRI may represent long term collateralisation of vessels and could represent the possibility of a "pseudo" single coronary anatomy. This was a limitation identified in using contrast imaging modalities and an autopsy (although not required) would be the only way to definitively exclude a chronic left system occlusion.

CTCA is a useful non-invasive imaging modality that is able to depict the origin and the course of coronary anomalies. Use of CTCA in combination with coronary angiography was useful in defining this unusual anatomy and excluding a haemodynamically significant lesion.

\section{References}

1. Siddiqui SM, Kesava RR, Kaza S, et al. Computed tomography coronary angiography diagnosis of single right coronary artery with congenital absence of left coronary artery system equivalents. Indian J Radiol Imaging. 2016 Apr-Jun; 26(2): 198-200.

2. Chuang CY, Chen YC, Cheng HS, et al. Congenital Anomaly of Single Dominant Right Coronary Artery with Hypoplastic Left Coronary Artery. Acta Cardiol Sin. 2015 Nov; 31(6): 557-9.

3. Lipton MJ, Barry WH, Obrez I, et al. Isolated Single Coronary Artery: Diagnosis, Angiographic Classification, and Clinical Significance. Radiology. 1979 Jan; 130(1): 39-47. 